\title{
Preadmission usage of metformin and mortality in COVID-19 patients including the post-discharge period
}

\author{
Emre Sedar Saygili ${ }^{1}$ (1) $\cdot$ Ersen Karakiliç $^{1} \cdot$ Erdal Mert $^{2} \cdot$ Alper Şener $^{3} \cdot$ Arzu Mirci $^{4}$
}

Received: 24 September 2021 / Accepted: 21 October 2021 / Published online: 29 October 2021

(c) The Author(s), under exclusive licence to Royal Academy of Medicine in Ireland 2021

\begin{abstract}
Background The effect of preadmission metformin usage (PMU) on the mortality of coronavirus disease-2019 (COVID-19) patients with diabetes is conflicting. Most studies have focused on in-hospital mortality; however, mortality after discharge also increases in COVID-19 patients.

Aims Examining the effect of PMU on all-cause mortality, including the post-discharge period.

Methods Patients with diabetes who were hospitalised in 2020 due to COVID-19 were included in the study. They were divided into two groups: those with a history of metformin use $(\mathrm{MF}(+))$ and those without such history $(\mathrm{MF}(-))$. Propensity score matching (PSM) was performed at a ratio of 1:1 for age and sex. COX regression analyses were used to demonstrate risk factors for mortality.

Results We investigated 4103 patients hospitalised for COVID-19. After excluding those without diabetes or with chronic liver/kidney disease, we included the remaining 586 patients, constituting 293 women (50\%) with an overall mean age of $66 \pm 11.9$ years. After PSM analysis, the in-hospital and post-discharge mortality rates were higher in the MF( -$)$ group though not significantly different. However, overall mortality was higher in the MF( - ) group (51 (42.5\%) vs. 35 (29.2\%), $p=0.031$ ). For overall mortality, the adjusted HR was 0.585 (95\% CI: $0.371-0.920, p=0.020)$ in the MF( + ) group.

Conclusion PMU is associated with reducing all-cause mortality. This effect starts from the in-hospital period and becomes more significant with the post-discharge period. The main limitations were the inability to evaluate the compliance with metformin and the effects of other medications due to retrospective nature.
\end{abstract}

Keywords Coronavirus disease $2019 \cdot$ Metformin $\cdot$ Mortality $\cdot$ Preadmission $\cdot$ Treatment

\section{Background}

COVID-19 has a poor prognosis and the risk of death nearly doubles in people with diabetes mellitus (DM) [1-3]. Hypoglycaemic agents and diabetes itself can affect the course of Covid-19. Although the effects of antidiabetic drugs on

Emre Sedar Saygili

emre.saygili@comu.edu.tr

1 Department of Internal Medicine, Division of Endocrinology and Metabolism, Faculty of Medicine, Canakkale Onsekiz Mart University, Canakkale 17020, Turkey

2 Department of Internal Medicine, Faculty of Medicine, Canakkale Onsekiz Mart University, Canakkale, Turkey

3 Department of Infections Disease, Faculty of Medicine, Canakkale Onsekiz Mart University, Canakkale, Turkey

4 Department of Pulmonary Disease, Faculty of Medicine, Canakkale Onsekiz Mart University, Canakkale, Turkey
COVID-19 are not fully known, new studies are ongoing in this regard. Metformin is one of the most widely used oral hypoglycaemic agents. It also has immunomodulatory effects in animal models via the phosphorylating adenosine monophosphate-activated protein kinase [4]. In addition, some studies reveal that it could have an inhibitory effect, especially on some viruses, by improving insulin sensitivity [5].

Metformin has been used in the past to treat malaria and influenza [6]. Several studies reveal the beneficial clinical results of concomitant use of metformin in respiratory diseases, including chronic pulmonary diseases and pulmonary infection diseases [7-10]. In a comparative study, metformin significantly decreased the mortality rate of chronic lower respiratory tract diseases [11]. In a meta-analysis conducted before the COVID-19 era, five observational studies involving 1282 patients were examined, and preadmission usage of metformin was associated with low mortality in patients 
with diabetes and sepsis [12]. Given the high worldwide prevalence of type 2 diabetes and the increased risk of COVID-19-related deaths, it is crucial to investigate the safety of hypoglycaemic agents in COVID-19 patients with diabetes [13].

The effect of preadmission metformin usage in patients with COVID-19 was assessed using meta-analyses of various studies. Although most studies reveal results in favour of metformin, there were some methodological differences. Two of these studies employed the propensity score matching (PSM) method while forming the control group, thereby enhancing equality. Moreover, these studies did not evaluate post-discharge mortality data $[14,15]$. However, the increase in mortality continued after hospital discharge in COVID19 , with $10 \%$ rates reported within a few months after discharge $[16,17]$. This prompts the need for more studies to explore the effects of metformin as part of the COVID-19 treatment guidelines $[18,19]$.

Thus, we aimed at analysing the effect of preadmission usage of metformin on the mortality of COVID-19 by PSM, while including a post-discharge period of at least 90 days.

\section{Material-methods}

Our study is a retrospective cohort study. Included participants were patients with diabetes over 18 years of age, hospitalised at the Canakkale Onsekiz Mart University Medical Faculty Hospital with COVID-19 confirmed by radiological and/or PCR between March 12, 2020 and December 22, 2020. Information on the age, sex, medications, comorbidities, laboratory parameters and COVID19 severity were obtained from participants' hospital records. Patients with a history of chronic liver/kidney failure and pregnancy were excluded from the study [20]. The study was approved by the Local Ethics Committee of the Canakkale Onsekiz Mart University Medical School (2011-KAEK-27/2021-E.2100041806).

From the records, patients who were found to use metformin regularly in the last 6 months were considered to be using metformin before hospitalisation. They were divided into two groups: those who used metformin (MF) before hospitalisation $(\mathrm{MF}(+))$ and those who did not $(\mathrm{MF}(-))$. Individuals in the $\mathrm{MF}(+)$ group were propensity scorematched as 1:1 for age and sex to individuals in the MF(-) group. The patient recruitment scheme is outlined in Fig. 1 . The mortality status of the patients included in the study on March 22, 2021 (the first year of the first patient recruitment) was obtained from the Turkish Central Death Registry. Following this date, all cases included in the study had a mortality of at least 90 days. The primary endpoint of our study was at least 90 days of all-cause mortality. Secondary endpoints were in-hospital and post-discharge mortalities.

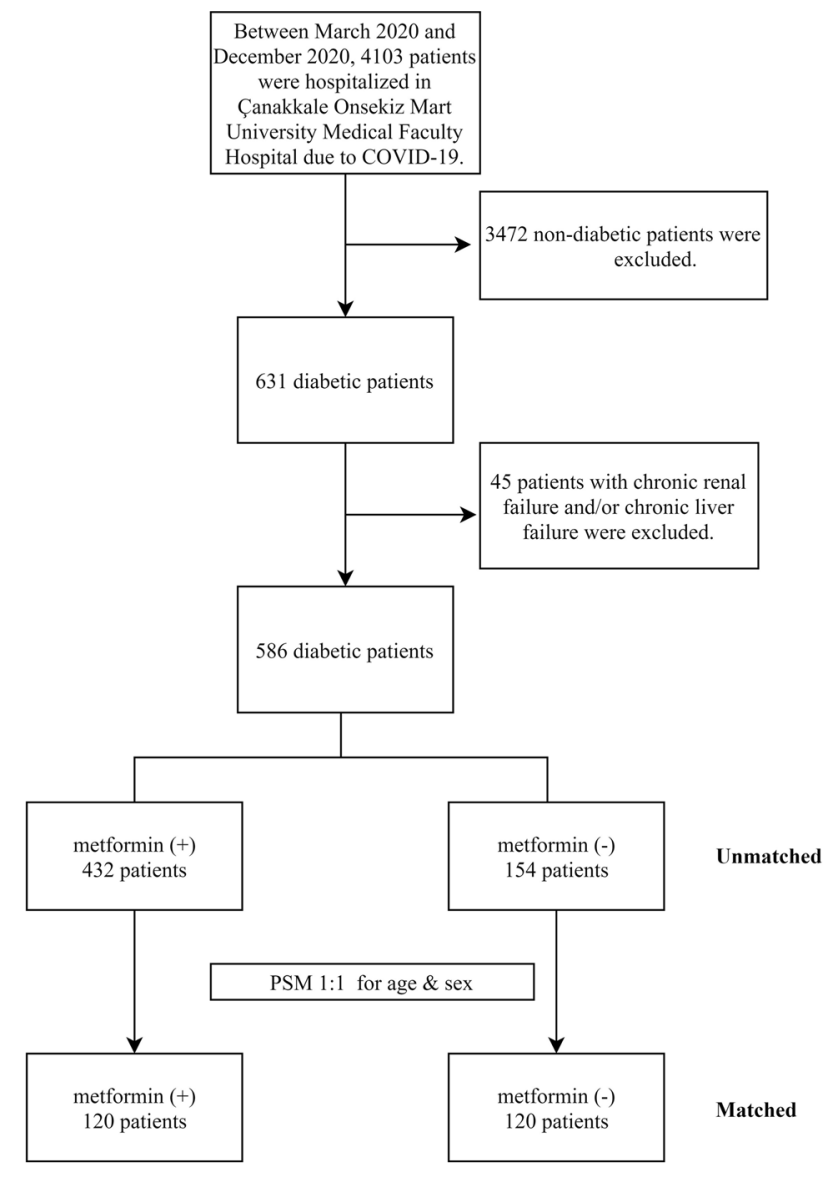

Fig. 1 Patient recruitment scheme

Normally distributed continuous variables are presented as mean \pm standard deviation, while non-normally distributed as the median and interquartile range (IQR: the difference between the 25th and 75th percentiles). Categorical variables were expressed as numbers and percentages. Student's $t$ test and Mann Whitney $U$ test were used to compare means between normally distributed and non-normally distributed groups of continuous variables, respectively. Pearson's chi-square was used to test the significance of the difference between categorical variables. Estimated glomerular filtration rate (eGFR) was calculated according to the CKD-EPI equation [21]. COX regression analyses were used to evaluate the risk factors of COVID-19-related mortality. In the adjusted HR (aHR) analysis, sex, age, glucose, ALT, eGFR, number of hospitalisation days, diabetes duration, chronic pulmonary disease, coronary artery disease, cerebrovascular disease, hypertension and hyperlipidaemia were used covariate variables. Unadjusted hazard ratios (HRs) were calculated by analysis with a single variable. Statistical analysis was performed with SPSS 19 for Windows (IBM, Armonk, NY, USA). A $p$-value $<0.05$ was considered statistically significant. 


\section{Results}

We included 586 diabetic patients, with a mean age of $66 \pm 11.9$ years, constituting 293 women (50\%). Metformin usage ratio was $73.7 \%$ (432).

\section{Analysis of data before PSM (unmatched)}

The mean age of the MF ( - ) group was significantly higher than the MF $(+)$ group $(68.92 \pm 13.47$ years vs $64.98 \pm 11.19$ years, $p=0.001)$. The MF $(-)$ group had lower baseline eGFR values $(62.2(35.5-87.9)$ vs. $76.5(56.8-94.4), p=0.001)$ and higher acute renal failure rates $(39 \%$ vs $19.4 \%, p=0.0001)$. Median number of hospitalization days (IQR) $(8(5-16)$ vs. $6(5-12), p=0.036)$, intensive care unit (ICU) admission rate $(27.3 \%$ vs. $18.1 \%$, $p=0.015)$, in-hospital mortality rate $(26 \%$ vs. $11.6 \%$, $p=0.0001)$, post-discharge mortality rate $(20.2 \%$ vs. $9.4 \%$, $p=0.002)$, and overall mortality rate $(40.9 \%$ vs. $19.9 \%$, $p=0.0001)$ ) were higher in the $\mathrm{MF}(-)$ group. Comparison of general characteristics of $\mathrm{MF}(+)$ and $\mathrm{MF}(-)$ groups before PSM is given in Table 1.

\section{Analysis of data after PSM (matched)}

PSM was performed at a ratio of 1:1 in terms of age and sex, according to the use of metformin. After PSM, 120
$\mathrm{MF}(+)$ and $120 \mathrm{MF}(-)$ patients were matched. ALT values (median(IQR)) of the MF $(+)$ group were higher than those of the MF (-) group (25(16-42) vs 19(11-30), $p=0.003)$. ICU admission rates, in-hospital mortality rates, and postdischarge mortality rates were higher in the $\mathrm{MF}(-)$ group, though not significant. Overall mortality was higher in the $\mathrm{MF}(-)$ group than in the $\mathrm{MF}(+)$ group (51(42.5\%) vs. $35(29.2 \%), p=0.031)$. A comparison of general characteristics of MF (+) and MF ( - ) groups after PSM is presented in Table 2.

\section{Hazard ratios for overall, in-hospital mortality, and post-discharge mortality}

HR for in-hospital mortality, post-discharge mortality, and overall mortality were evaluated with COX regression in matched and unmatched groups.

For overall mortality, the aHR was 0.607 (95\% CI: $0.428-0.860, p=0.005)$ in the unmatched $\mathrm{MF}(+)$ group, while the aHR was 0.585 (95\% CI: $0.371-0.920, p=0.020$ ) in the matched $\mathrm{MF}(+)$ group. For in-hospital mortality, aHR was 0.546 (95\% CI: $0.349-0.855, p=0.008)$ in the unmatched $\mathrm{MF}(+)$ group, while aHR was $0.568(95 \%$ CI: $0.306-1.052, p=0.072)$ in the matched $\mathrm{MF}(+)$ group. For post-discharge mortality, aHR was $0.702(95 \%$ CI:0.399-1.236, $p=0.22)$ in the unmatched $\operatorname{MF}(+)$ group and 0.652 (95\% CI:0.324 - 1.310, $p=0.23)$ in the matched $\mathrm{MF}(+)$ group. All mortality assessments, HR and
Table 1 Comparison of general characteristics of $\mathrm{MF}(+)$ and $\mathrm{MF}(-)$ groups before PSM

\begin{tabular}{llll}
\hline & MF(+) & MF( -$)$ & $p$ \\
\hline Age & $64.98 \pm 11.19$ & $68.92 \pm 13.47$ & $\mathbf{0 . 0 0 1}$ \\
Male (\%) & $215(49.8)$ & $217(50.2)$ & 0.85 \\
Diabetes duration above 10 years (\%) & $175(40.5)$ & $74(48.1)$ & 0.104 \\
Glucose (median (IQR)) & $191(136-267)$ & $198.5(152-280)$ & 0.08 \\
eGFR(median (IQR)) & $76.5(56.8-94.4)$ & $62.2(35.5-87.9)$ & $\mathbf{0 . 0 0 1}$ \\
ALT (median (IQR)) & $22(13-32)$ & $21(13-31)$ & 0.069 \\
HBA1C (median (IQR)) & $8(6.8-9.9)$ & $7.7(6.7-10.1)$ & 0.526 \\
Hypertension (\%) & $290(67.1)$ & $108(70.1)$ & 0.493 \\
COPD-asthma (\%) & $63(14.6)$ & $30(19.5)$ & 0.153 \\
CAD (\%) & $143(33.1)$ & $62(40.3)$ & 0.11 \\
CVD(\%) & $37(8.6)$ & $16(10.4)$ & 0.498 \\
Hyperlipidaemia (\%) & $179(41.4)$ & $51(33.1)$ & 0.07 \\
ARF(\%) & $84(19.4)$ & $60(39.0)$ & $\mathbf{0 . 0 0 0 1}$ \\
ICU admission (\%) & $78(18.1)$ & $42(27.3)$ & $\mathbf{0 . 0 1 5}$ \\
In-hospital mortality(\%) & $50(11.6)$ & $40(26.0)$ & $\mathbf{0 . 0 0 0 1}$ \\
Post-discharge mortality (\%) & $36(9.4)$ & $23(20.2)$ & $\mathbf{0 . 0 0 2}$ \\
Overall mortality (\%) & $86(19.9)$ & $63(40.9)$ & $\mathbf{0 . 0 0 0 1}$ \\
Number of hospitalization days (median(IQR)) & $6(5-12)$ & $8(5-16)$ & $\mathbf{0 . 0 3 6}$ \\
Follow-up days count & $157.5(105-235.7)$ & $127.5(34.2-212.7)$ & $\mathbf{0 . 0 0 1}$ \\
\hline
\end{tabular}

$C O P D$ chronic obstructive pulmonary disease, $C A D$ coronary artery disease, $C V D$ cerebrovascular disease, $A R F$ acute renal failure, $I C U$ intensive care unit, $e G F R$ estimated glomerular filtration rate 
Table 2 Comparison of general characteristics of $\mathrm{MF}(+)$ and $\mathrm{MF}(-)$ groups after PSM

\begin{tabular}{llll}
\hline & $\mathrm{MF}(+)$ & $\mathrm{MF}(-)$ & $\mathrm{p}$ \\
\hline Age & $68.72+10.04$ & $68.72+10.04$ & 1 \\
Male (\%) & $63(52.5)$ & $63(52.5)$ & 1 \\
Diabetes duration above 10 years (\%) & $64(53.3)$ & $56(46.7)$ & 0.302 \\
Glucose (median (IQR)) & $175.2(134.5-251)$ & $190(138-259)$ & 0.507 \\
eGFR(median (IQR)) & $53.9(38.8-80.6)$ & $61.5(34.1-86.8)$ & 0.648 \\
ALT (median (IQR)) & $25(16-42)$ & $19(11-30)$ & $\mathbf{0 . 0 0 3}$ \\
HBA1C (median (IQR)) & $8.35(7.05-9.57)$ & $7.55(6.57-9.65)$ & 0.214 \\
Hypertension (\%) & $89(74.2)$ & $88(73.3)$ & 0.883 \\
COPD-asthma (\%) & $20(16.7)$ & $25(20.8)$ & 0.408 \\
CAD (\%) & $48(40.0)$ & $50(41.7)$ & 0.793 \\
CVD(\%) & $15(12.5)$ & $11(9.2)$ & 0.406 \\
Hyperlipidaemia (\%) & $51(42.5)$ & $39(32.5)$ & 0.110 \\
ARF(\%) & $55(45.8)$ & $51(42.5)$ & 0.603 \\
ICU Admission (\%) & $23(19.2)$ & $35(29.2)$ & 0.07 \\
In-hospital mortality (\%) & $19(15.8)$ & $29(24.2)$ & 0.107 \\
Post-discharge mortality (\%) & $16(15.8)$ & $22(24.2)$ & 0.148 \\
Overall mortality (\%) & $35(29.2)$ & $51(42.5)$ & $\mathbf{0 . 0 3 1}$ \\
Number of hospitalization days (median (IQR)) & $7(5-12.5)$ & $8.5(5-16.5)$ & 0.071 \\
Follow-up days count & $140(88.7-213.7)$ & $127.5(39.7-211.5)$ & 0.216 \\
\hline COPD chric & &
\end{tabular}

$C O P D$ chronic obstructive pulmonary disease, $C A D$ coronary artery disease, $C V D$ cerebrovascular disease, $A R F$ acute renal failure, $I C U$ intensive care unit, $e G F R$ estimated glomerular filtration rate
aHR ratios in the match and unmatch groups, and forest plot graphics are given in Fig. 2. Figure 3 displays the Kaplan - Meier plot showing the risk of overall mortality in the unmatched and matched groups for $\operatorname{MF}(+)$ vs. $\operatorname{MF}(-)$ patients.

In the COX regression analysis performed for overall mortality, in addition to metformin, age (95\% CI: $1.003-1.057$, aHR; $1.029, p=0.031)$, ALT $(95 \% \mathrm{CI}$ : $1.000-1.001$, aHR; $1.001, p=0.001)$, and eGFR (95\% CI: $0.971-0.990$, aHR; $0.98, p=0.001)$ remained statistically significant.

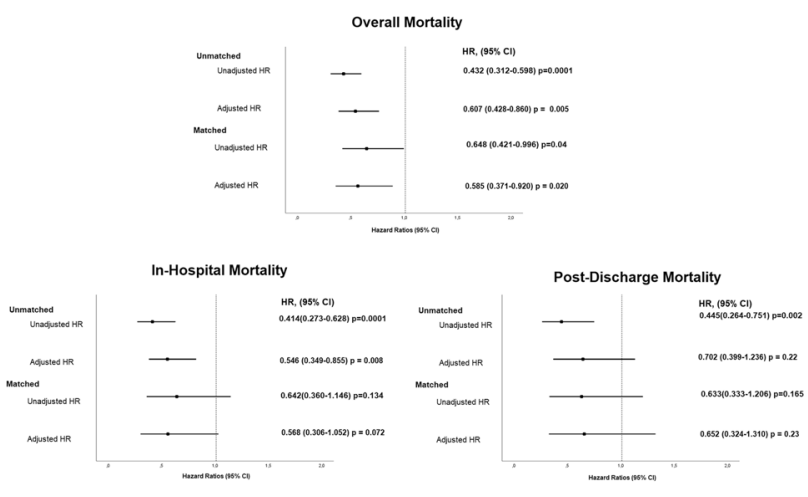

Fig. 2 Forest plot showing hazard ratios (HRs) of overall, in-hospital, post-discharge mortality for MF (+) compared to MF (-) patients

\section{Discussion}

Although diabetes is a major risk factor for COVID-19 mortality, the safe use of hypoglycaemic agents in glycaemic control in such patients during the COVID-19 pandemic was unclear $[2,22]$. Our study showed that the usage of metformin before hospital admission had a protective effect on mortality. This effect increases over time and becomes more significant on inclusion of the postdischarge period. In our study, the effect remained after PS matching for age and sex. Moreover, after adjusting for comorbidities, metformin provides a decrease in the mortality risk of approximately $45 \%$. Having post-discharge follow-ups distinguishes our study from other related studies, thereby contributing to the literature.

When in-hospital mortality was evaluated, the protectiveness for HR and aHR mortality in the $\mathrm{MF}(+)$ group became insignificant after grouped HR matching. Only unmatched HR was protective for mortality in the $\mathrm{MF}(+)$ group for the post-discharge period. However, both unmatch and match HR and aHR rates were protective for overall mortality in the $\mathrm{MF}(+)$ group.

A recent meta-analysis examining the association between metformin use and overall COVID-19 mortality found an OR of 0.78 (95\% CI $0.71-0.86)$ with PSM studies and 0.75 (95\% CI 0.66-0.85) for unmatched studies [23]. The approximately $25 \%$ risk reduction found in 

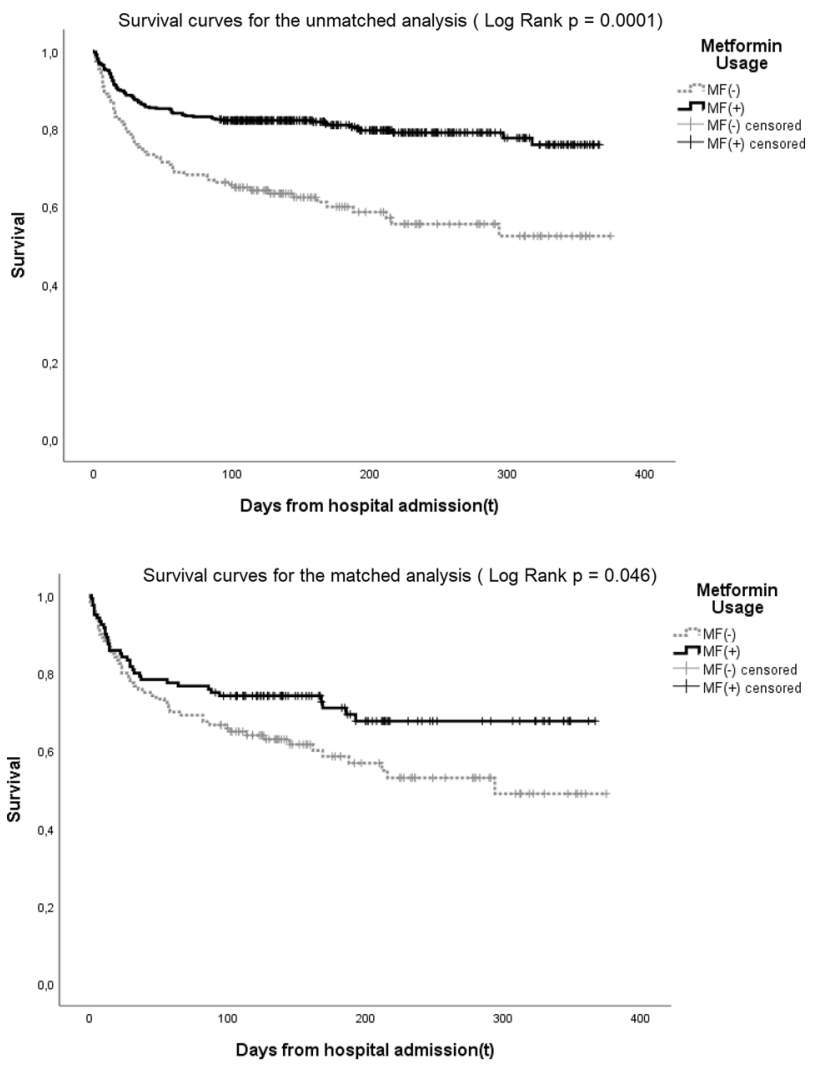

Fig. 3 Kaplan-Meier plot showing the risk of overall mortality in the unmatched and matched groups for MF (+) vs. MF (-) patients

this meta-analysis was approximately $40 \%$ in our study. The fact that we have follow-ups after discharge, our long follow-up period and the inclusion of only hospitalised patients in the study may be the factors affecting this difference.

A meta-analysis in 2020 evaluated nine studies on metformin and COVID-19 hospital mortality. The OR for the non-adjusted model was 0.45 [95\% CI: $0.25-0.81$ ], and that for the adjusted model was 0.64 [95\% CI: 0.43, 0.97] [24]. However, PSM was not performed in some of the studies evaluated in this meta-analysis. In our study, the preadmission usage of metformin was observed to be protective on in-hospital mortality before PSM, but not after PSM.

Metformin is contraindicated for patients with diabetes having comorbidities such as chronic kidney disease, which has a high risk for mortality. Therefore, metforminusable patients have a lower diabetic burden [25]. The exclusion of this group in our study provided a more accurate interpretation of the metformin effect. However, some studies did not exclude chronic kidney disease in their analysis [26-28]. In a population-based study involving primary care patients, preadmission usage of metformin had no protective effect compared to in-hospital studies
[29]. This could be due to the exclusion of the lower risk group. A recent large cohort exploring the registry containing all patients with diabetes nationwide (in-hospital and out-hospital data) also had results consistent with our study. This study showed that patients on metformin prior to infection had a lower risk of death associated with COVID-19 with an HR of 0.77 (95\% CI 0.73-0.81) [13]. Although studies on the COVID-19 mortality of preadmission usage of metformin have different results, the differences in the selection of patient and control groups could impact the results.

Although both groups' median ALT levels were within the normal reference range, ALT levels were higher in metformin users. In our study, higher ALT levels were associated with mortality. Other studies have also found that ALT can predict increased mortality [30, 31]. Despite the higher ALT levels in the metformin group, a lower mortality rate may support the protective effect of metformin in our study.

Our study has some limitations. Since the deaths of the patients after discharge were evaluated with the national death registration system, the death status at home or in another hospital could also be determined precisely from the system. However, since COVID patients who were hospitalised were evaluated in our study, patients who died before hospitalisation due to peri-hospital cardiac arrest could not be evaluated within the scope of this study. Even though we observed in the prescription records that eligible participants were on drugs, it is possible that the patients were not compliant to their medications. In addition, we evaluated the just efficacy of metformin, not the consequences, complications, or interactions of other hypoglycaemic agents and non-diabetic medications that can be used in combination therapy in diabetes patients. Furthermore, the causality could not be inferred from our observations owing to the study design. Randomised controlled studies are needed to evaluate the mentioned effects more clearly. However, these studies are still ongoing. Although studies show beneficial effects, if the patient had impaired renal/hepatic function, discontinuation of this drug is recommended if there are concerns about acidosis.

\section{Conclusion}

Preadmission use of metformin is associated with reducing all-cause mortality following hospitalisation for COVID-19. This effect starts from the in-hospital period, but it becomes significant when evaluated within the post-discharge period. Considering that patients with diabetes have a higher risk of death after COVID-19, this positive effect of metformin is reassuring for clinicians and patients. 
Acknowledgements We acknowledge and are grateful to all health care professionals at Canakkale Onsekiz Mart University, who cared for the patients during the COVID-19 pandemic. Preparation for publication of this article is supported by the Society of Endocrinology and Metabolism of Turkey.

Data availability The data of the study are available from the corresponding author when a reasonable request.

\section{Declarations}

Ethics approval The study was approved by the Local Ethics Committee of the Canakkale Onsekiz Mart University Medical School (2011-KAEK-27/2021-E.2100041806).

Conflict of interest The authors declare no competing interests.

\section{References}

1. Apicella M, Campopiano MC, Mantuano M et al (2020) COVID19 in people with diabetes: understanding the reasons for worse outcomes. Lancet Diabetes Endocrinol 8:782-792. https://doi.org/ 10.1016/S2213-8587(20)30238-2

2. Barron E, Bakhai C, Kar P et al (2020) Associations of type 1 and type 2 diabetes with COVID-19-related mortality in England: a whole-population study. Lancet Diabetes Endocrinol 8:813-822. https://doi.org/10.1016/S2213-8587(20)30272-2

3. Guo W, Li M, Dong Y et al (2020) Diabetes is a risk factor for the progression and prognosis of COVID -19. Diabetes Metab Res Rev 36:e3319. https://doi.org/10.1002/dmrr.3319

4. Kajiwara C, Kusaka Y, Kimura S et al (2018) Metformin mediates protection against Legionella pneumonia through activation of AMPK and mitochondrial reactive oxygen species. J Immunol 200:623-631. https://doi.org/10.4049/jimmunol.1700474

5. Chen Y, Gu F, Guan J-L (2018) Metformin might inhibit virus through increasing insulin sensitivity. Chin Med J (Engl) 131:376-377. https://doi.org/10.4103/0366-6999.223856

6. Bailey CJ (2017) Metformin: historical overview. Diabetologia 60:1566-1576. https://doi.org/10.1007/s00125-017-4318-z

7. Ho TW,Huang CT, Tsai YJ et al (2019) Metformin use mitigates the adverse prognostic effect of diabetes mellitus in chronic obstructive pulmonary disease Respir Res 20https://doi.org/10. 1186/s12931-019-1035-9

8. Wu TD, Keet CA, Fawzy A et al (2019) Association of metformin initiation and risk of asthma exacerbation A claims-based cohort study. Ann Am Thorac Soc 16:1527-1533. https://doi.org/10. 1513/AnnalsATS.201812-8970C

9. Mortensen E, Anzueto A (2018) Association of metformin and mortality for patients with diabetes who are hospitalised with pneumonia. In: Respiratory infections. European Respiratory Society, p PA2639

10. Zhang M, He J (2020) Impacts of metformin on tuberculosis incidence and clinical outcomes in patients with diabetes: a systematic review and meta-analysis. Eur J Clin Pharmacol 76:149-159. https://doi.org/10.1007/s00228-019-02786-y

11. Mendy A, Gopal R, Alcorn JF, Forno E (2019) Reduced mortality from lower respiratory tract disease in adult diabetic patients treated with metformin. Respirology 24:646-651. https://doi.org/ 10.1111/resp. 13486

12. Liang H, Ding X, Li L et al (2019) Association of preadmission metformin use and mortality in patients with sepsis and diabetes mellitus: a systematic review and meta-analysis of cohort studies. Crit Care 23:50. https://doi.org/10.1186/s13054-019-2346-4

13. Khunti K, Knighton P, Zaccardi F et al (2021) Prescription of glucose-lowering therapies and risk of COVID-19 mortality in people with type 2 diabetes: a nationwide observational study in England. Lancet Diabetes Endocrinol 9:293-303. https://doi. org/10.1016/S2213-8587(21)00050-4

14. Hariyanto TI, Kurniawan A (2020) Metformin use is associated with reduced mortality rate from coronavirus disease 2019 (COVID-19) infection. Obes Med 19:100290. https://doi.org/10. 1016/j.obmed.2020.100290

15. Kow CS, Hasan SS (2021) Mortality risk with preadmission metformin use in patients with COVID-19 and diabetes: a metaanalysis. J Med Virol 93:695-697. https://doi.org/10.1002/jmv. 26498

16. Donnelly JP, Wang XQ, Iwashyna TJ, Prescott HC (2021) Readmission and death after initial hospital discharge among patients with COVID-19 in a large multihospital system. JAMA 325:304. https://doi.org/10.1001/jama.2020.21465

17. Ayoubkhani D, Khunti K, Nafilyan V et al (2021) Post-covid syndrome in individuals admitted to hospital with covid-19: retrospective cohort study. BMJ 372 https://doi.org/10.1136/ bmj.n693

18. Do JY, Kim SW, Park JW et al (2020) Is there an association between metformin use and clinical outcomes in diabetes patients with COVID-19? Diabetes Metab 101208https://doi.org/10. 1016/j.diabet.2020.10.006

19. Zangiabadian M, Nejadghaderi SA, Zahmatkesh MM et al (2021) The efficacy and potential mechanisms of metformin in the treatment of COVID-19 in the diabetics: a systematic review Front Endocrinol (Lausanne) $12 \mathrm{https} / / /$ doi.org/10.3389/fendo.2021. 645194

20. Cheng X, Liu Y-M, Li H et al (2020) Metformin is associated with higher incidence of acidosis, but not mortalitY, in individuals with COVID-19 and pre-existing type 2 diabetes. Cell Metab 32:537547.e3. https://doi.org/10.1016/j.cmet.2020.08.013

21. Levey AS, Stevens LA, Schmid CH et al (2009) A new equation to estimate glomerular filtration rate. Ann Intern Med 150:604-612. https://doi.org/10.7326/0003-4819-150-9-200905050-00006

22. Ceriello A, Stoian AP, Rizzo M (2020) COVID-19 and diabetes management: what should be considered? Diabetes Res Clin Pract 163:108151. https://doi.org/10.1016/j.diabres.2020.108151

23. Avogaro A, Bonora B, Fadini GP (2021) Managing diabetes in diabetic patients with COVID: where do we start from? Acta Diabetol. https://doi.org/10.1007/s00592-021-01739-1

24. Lukito AA, Pranata R, Henrina J et al (2020) The effect of metformin consumption on mortality in hospitalized COVID-19 patients: a systematic review and meta-analysis. Diabetes Metab Syndr Clin Res Rev 14:2177-2183. https://doi.org/10.1016/j.dsx. 2020.11.006

25. Dave JA, Tamuhla T, Tiffin N et al (2021) Risk factors for COVID19 hospitalisation and death in people living with diabetes: a virtual cohort study from the Western Cape Province South Africa Diabetes Res Clin Pract 108925 https://doi.org/10.1016/j.diabres. 2021.108925

26. Luo P, Qiu L, Liu Y et al (2020) Metformin treatment was associated with decreased mortality in COVID-19 patients with diabetes in a retrospective analysis. Am J Trop Med Hyg 103:69-72. https://doi.org/10.4269/ajtmh.20-0375

27. Ghany R, Palacio A, Dawkins E et al (2021) Metformin is associated with lower hospitalisations, mortality and severe coronavirus infection among elderly medicare minority patients in 8 states in USA. Diabetes Metab Syndr Clin Res Rev 15:513-518. https:// doi.org/10.1016/j.dsx.2021.02.022 
28. Chen Y, Yang D, Cheng B et al (2020) Clinical characteristics and outcomes of patients with diabetes and COVID-19 in association with glucose-lowering medication. Diabetes Care 43:1399-1407. https://doi.org/10.2337/dc20-0660

29. Wang J, Cooper JM, Gokhale K et al (2021) Association of metformin with susceptibility to COVID-19 in people with type 2 diabetes. J Clin Endocrinol Metab 106:1255-1268. https://doi. org/10.1210/clinem/dgab067

30. Salık F, Uzundere O, Bıçak M et al (2021) Liver function as a predictor of mortality in COVID-19 Retrospective study Ann Hepatol 100553https://doi.org/10.1016/j.aohep.2021.100553
31. Medetalibeyoglu A, Catma Y, Senkal N et al (2019) The effect of liver test abnormalities on the prognosis of COVID-19. Ann Hepatol 19:614-621. https://doi.org/10.1016/j.aohep.2020.08.068

Publisher's Note Springer Nature remains neutral with regard to jurisdictional claims in published maps and institutional affiliations. 